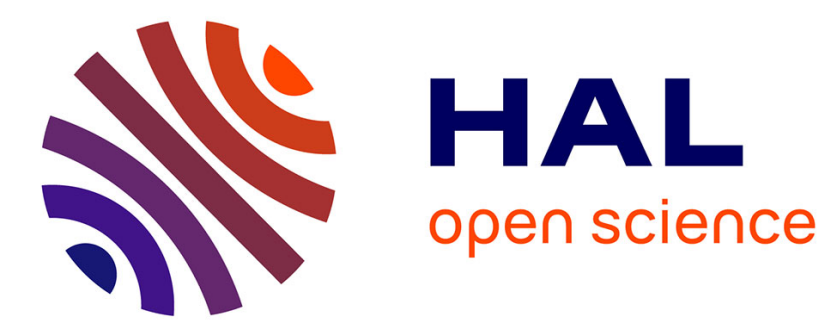

\title{
Defect pairs and clusters related to the EL2 centre in GaAs
}

\author{
Sherif Makram-Ebeid, P. Boher
}

\section{To cite this version:}

Sherif Makram-Ebeid, P. Boher. Defect pairs and clusters related to the EL2 centre in GaAs. Revue de Physique Appliquée, 1988, 23 (5), pp.847-862. 10.1051/rphysap:01988002305084700 . jpa-00245888

\section{HAL Id: jpa-00245888 https://hal.science/jpa-00245888}

Submitted on 1 Jan 1988

HAL is a multi-disciplinary open access archive for the deposit and dissemination of scientific research documents, whether they are published or not. The documents may come from teaching and research institutions in France or abroad, or from public or private research centers.
L'archive ouverte pluridisciplinaire HAL, est destinée au dépôt et à la diffusion de documents scientifiques de niveau recherche, publiés ou non, émanant des établissements d'enseignement et de recherche français ou étrangers, des laboratoires publics ou privés. 


\title{
Defect pairs and clusters related to the EL2 centre in GaAs
}

\author{
Sherif Makram-Ebeid and P. Boher \\ Laboratoires d'Electronique et de Physique Appliquée (*), 3 avenue Descartes, F-94450 Limeil-Brévannes, \\ France
}

(Reçu le 15 septembre 1987, accepté le 16 novembre 1987)

\begin{abstract}
Résumé. - Dans cet article, nous proposons des interprétations cohérentes des données expérimentales sur l'As ${ }_{\mathrm{Ga}}$ irradié obtenues par des études de spectroscopie de transitoires de niveaux profonds (DLTS), de résonance paramagnétique électronique et de conductivité électrique. Notre analyse révèle un niveau donneur simple (MG) situé au milieu de la bande interdite et apparenté à l'antisite $\mathrm{As}_{\mathrm{Ga}}$. Nous fournissons des arguments permettant d'identifier le niveau MG avec celui du centre EL2 natif du matériau massif. Nous nous servons d'un modèle physique récemment mis au point pour le calcul des taux de transition électronique tunnel assisté phonon entre défauts. Dans les matériaux irradiés à forte dose on peut attribuer la conductivité électrique à des sauts d'électrons entre défauts MG. Nos calculs sont en parfait accord avec les résultats expérimentaux de Deng et al. pour la conductivité en courant continu de l'As $\mathrm{Ga}_{\mathrm{Ga}}$ induite par un bombardement aux ions d'argon et ceci pour une gamme de températures allant de $20 \mathrm{~K}$ environ à $500 \mathrm{~K}$. En cherchant un accord entre calculs et observations, on trouve une énergie de phonon ( $\hbar \omega=20 \pm 2 \mathrm{meV}$ ) et une énergie Franck-Condon $(S \hbar \omega=145 \pm 10 \mathrm{meV})$ pour MG qui sont pratiquement identiques à celles déterminées au préalable pour EL2. La conductivité pour courants alternatifs a aussi été calculée et se trouve en accord avec les résultats expérimentaux de Mareš et al. pour l'As ${ }_{\mathrm{Ga}}$ semi-isolant de haute pureté. A basses fréquences, nous pouvons attribuer complètement cette conductivité à une répartition homogène et aléatoire de défauts EL2 dans le volume. Pour rendre compte des résultats DLTS, nous sommes conduits à invoquer le niveau MG (identifiable à celui de EL2). Pour l'As la $_{\mathrm{Ga}}$ de type $\mathrm{n}$ irradié aux électrons rapides, les pics E4 et E5 apparaissant vers les hautes températures des spectres DLTS sont attribués à deux paires de défauts comprenant chacune un niveau (MG). Pour les matériaux $\mathrm{As}_{\mathrm{Ga}}$ de type $\mathrm{n}$ irradiés aux particules lourdes, des amas de défauts rendent compte des spectres DLTS. Nos simulations pour ces paires et amas prévoient correctement les positions des pics DLTS et leur dépendance du champ électrique.
\end{abstract}

\begin{abstract}
In this article, we attempt to consistently interpret Deep Level Transient Spectroscopy (DLTS), Electron Paramagnetic Resonance (EPR) and electrical conductivity experimental data for irradiated GaAs. Our analysis reveals the occurrence of an irradiation-induced single donor mid-gap level (MG) related to the $\mathrm{As}_{\mathrm{Ga}}$ antisite defect. We give evidence that MG can be identified with the deep level associated to the native bulk centre EL2. We make use of a model we have recently developed for the calculation of inter-defect phonon-assisted tunnelling rates. We calculate the nearest neighbour defect-induced hopping conductivities. In heavy dose irradiated GaAs, the DC conductivity is attributed to hopping between MG defects. Our calculations provide extremely good fits to the experimental results of Deng et al. for the DC electrical conductivity in GaAs induced by argon ion bombardment for temperatures ranging from around $20 \mathrm{~K}$ to $500 \mathrm{~K}$. The phonon energy $\hbar \omega(20 \pm 2 \mathrm{meV})$ and Franck-Condon shift $S \hbar \omega(145 \pm 10 \mathrm{meV})$ of MG needed to achieve this fit are in very good agreement with the $\hbar \omega$ and $S \hbar \omega$ values earlier determined for the native EL2 defect. The bulk AC hopping conductivity has also been calculated and found to agree well with experimental results of Mareš et al. for high purity semi-insulating GaAs. The corresponding low-frequency AC conductivity is attributed entirely to a homogeneous random distribution of native mid-gap donor defects EL2. We are also led to invoke the mid-gap level MG (identifiable with that of EL2) in order to account for DLTS results. In fast-electron irradiated n-GaAs, the two high temperature DLTS peaks E4 and E5 are both accounted for by two defect pairs each including an MG level component. In heavy-particle bombarded n-GaAs damage clusters including MG defects account for the experimentally recorded high temperature DLTS peak. For these defect pairs and clusters, our DLTS simulations correctly predict the observed peak positions and their dependence on the electric field.
\end{abstract}

(*) Laboratoires d'Electronique et de Physique Appliquée (LEP) is a member of the Philips Research Organisation. 


\section{Introduction.}

The EL2 deep-level defect in GaAs is of great practical importance because it is essential in pinning the Fermi level near mid-gap in high purity and in indium-doped semi-insulating substrates. This has stimulated an abundant literature over the properties of EL2 [1, 2] and over its identification (see e.g. Refs. [1-5]). The symmetry and paramagnetic optically monitored - resonance behaviour indicate that it is a complex including the antisite defect $\mathrm{As}_{\mathrm{Ga}}$. Recent publications [5] support the attribution of EL2 to a second-neighbour complex $\left(\mathrm{As}_{\mathrm{Ga}} \mathrm{As}_{\mathrm{i}}\right)$ with an arsenic interstetial atom as earlier suggested by von Bardeleben et al. [4].

The Fermi-level has been reported to be pinned near mid-gap in GaAs materials after a large dose of fast-electron [4] or heavy-particle (fast-neutron or ion) $[6,7]$ bombardment. A similar Fermi-level pinning is also observed near real GaAs surfaces [8] irrespective of the surface treatment or dielectric deposition used. In all these cases, a defect compensation mechanism including a mid-gap level is responsible for the Fermi-level pinning. This is very similar to the compensation occuring in undoped or indium doped semi-insulating GaAs substrates and where EL2 is involved.

In this article we attempt to decide whether the mid-gap level induced by electron or heavy-particle irradiation is related to the native EL2 level. As will be seen later, this irradiation-induced mid-gap level systematically appears to be part of a close defect pair or of a dense damage cluster. Electron hopping probabilities between defects in these pairs and clusters cannot be neglected and prevent a straightforward interpretation of the electrical properties of irradiated GaAs.

In order to gain insight into these problems, we make use of our newly developed theory for the practical calculation of multiphonon tunnelling transition rates between deep level defect states in a semiconductor [9]. The fact that multiphonon effects may assist the ionization of isolated defects in such materials has earlier been demonstrated $[10,11]$. We use our theory for predicting the defect-induced DC and AC electrical conductivities as well as the DLTS behaviour. We compare our theoretical simulations with experimental results.

\section{Evaluation of multiphonon hopping rates.}

In this section we describe the method we adopted for the evaluation of the inter-defect multiphonon tunnelling rates. The corresponding theory was recently developed [9].

Two defects labelled $i=1$ and 2 are assumed to interact and their centroids to be a distance $r_{12}$ apart. An electric field of intensity $F$ acts at an angle $\theta$ with the line joining the defect centroids. We calculate the electronic terms by making use of a Quantum Defect Model (QDM) [12]. In such a model, the potential of defect $i$ at distances larger than a value $a_{i}$ from its centroid is simply taken to be the long range Coulomb potential $\varphi_{i}(\mathbf{r})$ given by

$$
\varphi_{i}(\mathbf{r})=-\frac{\alpha_{i}}{\left|\mathbf{r}-\mathbf{r}_{i}\right|}
$$

with

$$
\alpha_{i}=q^{2} Z_{i} /\left(4 \pi \varepsilon_{0} \varepsilon_{r}\right)
$$

where $\mathbf{r}_{i}$ is the positional radius vector for the centroid of defect $i, \mathbf{r}$ is the positional vector of an arbitrary point in the semiconductor and $a_{i}, Z_{i} q$ stands for the total charge of the ionized defect, $q$ is the elementary charge and $\varepsilon_{0} \varepsilon_{r}$ the dielectric constant of the semiconductor. The numerical value of $\alpha_{i}$ for $\operatorname{GaAs}\left(\varepsilon_{r}=12.53\right)$ is about $1.15 \mathrm{eV} \AA$ for a single donor.

The above long-range potential is assumed to hold in a region outside a sphere defined by $\left|\mathbf{r}-\mathbf{r}_{i}\right| \geqslant$ $a_{i}$. In this region, the semiconductor's energymomentum relationship is used to describe the electronic wave-function. Inside the sphere $\left|\mathbf{r}-\mathbf{r}_{i}\right|<a_{i}$ another (localized) Coulomb potential and a different electron effective mass have to be assumed and these are heuristically adjusted to agree with experimentally observed data of the defects. The radii $a_{i}$ are of the order of one or two lattice spacings.

In describing the long-range electron wave-functions (for $\left|\mathbf{r}-\mathbf{r}_{i}\right|>a_{i}$ ), we make use of Franz-Kane relationship [13] $K(\Delta)$ between the wave-function attenuation factor $K$ and the electronic energy $\Delta$ measured from the bottom of the conduction band. The electron wave function can thus be written as [10]

$$
\begin{array}{r}
\psi_{i}(\mathbf{r})=\eta_{i} \cdot \frac{\left(2 K_{i}\right)^{3 / 2}}{\left(4 \pi \Gamma\left(2 p_{i}+1\right)\right)^{1 / 2}} \cdot \frac{\mathrm{e}^{-A_{i}(\mathbf{r}) / 2}}{\left(2 K_{i}\left|\mathbf{r}-\mathbf{r}_{i}\right|\right)^{1-p_{i}}} \\
\text { for }\left|\mathbf{r}-\mathbf{r}_{i}\right|>a_{i}
\end{array}
$$

with $\quad K_{i}=K\left(\Delta_{i}\right), \quad p_{i}=\left.\alpha_{i} \frac{\mathrm{d} K(\Delta)}{\mathrm{d} \Delta}\right|_{\Delta=\Delta_{i}}$

and

$$
A_{i}(\mathbf{r})=2 \cdot \int_{0}^{1} K\left(\Delta_{i}-q \mathbf{F} \cdot\left(\mathbf{r}-\mathbf{r}_{i}\right) \zeta\right)\left|\mathbf{r}-\mathbf{r}_{i}\right| \mathrm{d} \zeta
$$

where we have assumed spherically symmetrical ( $s$ state) wave functions, $\Gamma(x)$ stands for the Gamma function [14], $\mathbf{F}$ is the electric field vector and $\Delta_{i}$ the energy of the localized electron measured below the conduction band. One recognizes the WKB expression $A_{i}(\mathbf{r})$ for the wave function attenuation. The numerical prefactor $\eta_{i}$ is such that $\left(1-\eta_{i}^{2}\right)$ is approximately the probability of finding the electron in the region $\left(\left|\mathbf{r}-\mathbf{r}_{i}\right| \leqslant a_{i}\right)$. For the deep-levels we are dealing with here, one expects a 
near unity probability for finding the electron inside the sphere $\left(\left|\mathbf{r}-\mathbf{r}_{i}\right| \leqslant a_{i}\right)$ and a hence small value of $\eta_{i}$. Such a strong localization of deep level wave functions had to be assumed both in reference [10], to account for electric field ionization of defects, and by Burt [15] to account for deep level photo-ionization cross-sections in GaAs.

In order for an electron to hop from one defect site to another, multi-phonon energy has to be exchanged with lattice-vibration modes. The defect labelled $i$ is assumed to be linearly coupled to a localized phonon mode of energy $\hbar \omega_{i}$ with a HuangRhys coupling constant $S_{i}$. One can then write the inter-defect hopping rate $R_{12}$ as an integral over all energies $E$ in the band-gap and for deep level defects, this takes the form [9]

$$
R_{12}=\int_{E} P(E) \cdot D_{1}\left(E-E_{T 1}\right) \cdot D_{2}\left(E_{T 2}-E\right) \cdot \mathrm{d} E
$$

where

$$
\begin{aligned}
P(E) \simeq \frac{256 \pi}{\hbar} \cdot \frac{\left(m^{*}\right)^{3 / 2}}{\left(m_{\mathrm{d} 1} m_{\mathrm{d} 2}\right)^{3 / 4}} \times & \\
& \times \eta_{1} \eta_{2}\left(\frac{E_{\mathrm{co}}-E}{A(E)}\right)^{2} \mathrm{e}^{-A(E)}
\end{aligned}
$$

with

$$
\begin{array}{r}
A(E)=2 \int_{-1}^{+1} K\left(E_{\mathrm{co}}-E-q F \cdot \cos \theta \cdot \frac{r_{12}}{2} \cdot \zeta\right) \times \\
\times r_{12} \cdot \mathrm{d} \zeta
\end{array}
$$

where $E_{\mathrm{co}}$ is the conduction-band energy mid-way between the two defects, $K(\Delta)$ stands for the FranzKane dispersion relation $m^{*}$ and $m_{\mathrm{d} i}$ are the effective mass in the conduction band and near the defect centroids respectively, $E_{T 1}$ and $E_{T 2}$ are the total (electron + lattice) free energy before and after the hopping transition respectively. The function $D_{i}\left(E_{p}\right)$ represents the probability density (per unit energy) that an electron trapped in defect $i$ will absorb a multiphonon energy $E_{p}$. Negative $E_{p}$ values imply multiphonon emission. These functions (for $i=1$ and 2) are defined by

$$
\begin{aligned}
D_{i}\left(E_{p}\right)=\exp & \left(-\frac{E_{p}}{2 k T}-S_{i} \cdot \operatorname{coth}\left(\frac{\hbar \omega_{i}}{k T}\right)\right) \times \\
& \times I_{m}\left(\frac{S_{i}}{\sinh \left(\hbar \omega_{i} / 2 k T\right)}\right) / \hbar \omega_{i} .
\end{aligned}
$$

where $I_{m}$ is the modified Bessel function [14] of order $m$ and $m=-E_{p} / \hbar \omega_{i}$.

Reversing the roles of defects (1) and (2), the reverse tunnelling rate $R_{21}$ can be deduced. From the above results, it can be shown that

$$
R_{21}=R_{12} \cdot \exp \left(-\frac{E_{T 1}-E_{T 2}}{k T}\right)
$$

This can be also obtained as a result of the FermiDirac statistics from the principle of detailed balance.

In the above expression, when calculating the difference $\left(E_{T 1}-E_{T 2}\right)$ in total energy of the defects, one should, in general, include the effects of both the semiconductor electric field $\mathbf{F}$ and those of the long-range Coulomb potentials of the defects themselves. We then have

$$
E_{T 1}-E_{T 2}=\Delta_{T 2}-\Delta_{T 1}+q F \cdot r_{12} \cos \theta+\frac{\alpha_{1}-\alpha_{2}}{r_{12}}
$$

$\Delta_{T 1}$ and $\Delta_{T 2}$ being the ionization energies for isolated defects 1 and 2 respectively.

\section{Analysis of irradiation-induced defects in GaAs.}

In this and the following sections, we make use of the above results as a tool for interpreting experimental data related to the defects induced by fast electron, fast neutron irradiation and by ion implantation of GaAs.

Lang [16] has reported a comparison (made by Kimerling et al. at Bell Laboratories) between DLTS spectra obtained for $\mathrm{n}-\mathrm{GaAs}$ samples which have been previously bombarded by high energy particles of different types. The largest differences between these spectra appeared to be due to the deepest level (nearest to mid-gap). The corresponding DLTS peak becomes progressively wider when one goes from electron to proton, to $\mathrm{He}^{+}$and to $\mathrm{O}^{+}$irradiation. These authors attributed the difference in the spectra to the formation of defect clusters when heavier particles are used for bombarding the material. In contrast, electron bombardment is expected to produce simpler defects such as single vacancies, interstitials and Frenckel defect pairs. Physically, this is related to the fact that the mass of heavier ions is better matched than the mass of electrons to the lattice atoms. Hence, each impinging heavy-ion can impart a larger portion of its kinetic energy to lattice atoms. An initial interaction between an impinging ion and a lattice atom is referred to as a Primary Knock-On (PKO) event [17]. The lattice atom displaced during such an event can have considerable kinetic energy and is capable, in turn, of generating a cascade of other defects all localized within a small volume of the bombarded crystal. The geometry of the resulting damage clusters can be theoretically estimated [18] and is related to deposited energy data [19]. In the cases investigated here, the clusters are expected to have sizes of the order of $500 \AA$. When comparing DLTS results, fast-neutron-irradiated samples [20] are found to have spectra closer to those of heavy-ion implanted samples than to proton-bombarded samples [16]. This may seem surprising since protons and neutrons have practi- 
cally equal masses. It is, however, recalled that unlike protons, the interaction of neutrons with lattice atoms occurs exclusively through nuclear collisions (no electronic stopping) and hence neutrons generate clusters more efficiently than protons.

2.1 INDIVIDUAL DEFECTS INVOLVED. - We start by reviewing the defects observed in $\mathrm{n}-\mathrm{GaAs}$ after fast electron or gamma irradiation. The defects introduced by heavier particle bombardment are more difficult to analyse because of the occurrence of damage clusters as mentioned above.

2.1.1 Defects in fast-electron-irradiated n-GaAs. The main electron trapping levels observed for fastelectron irradiation have been labelled $[16,21] \mathrm{E} 1$ $\left(E_{\mathrm{c}}-0.045 \mathrm{eV}\right), \quad \mathrm{E} 2 \quad\left(E_{\mathrm{c}}-0.14 \mathrm{eV}\right)$ and $\mathrm{E} 3$ $\left(E_{\mathrm{c}}-0.310 \mathrm{eV}\right)$. These levels are the most extensively studied. Convincing evidence exists that they are all due to arsenic atom displacements and that $\mathrm{E} 1$ and E2 are related to two charge states of the same defect [21] (i.e. E1 = E2 + electron). Two other wide DLTS electron peaks labelled E4 and E5 are also observed with appreciable amplitudes. The introduction rates of E1, E2, E3, E4 and E5 in $\mathrm{n}-\mathrm{GaAs}$ for $1 \mathrm{MeV}$ electron irradiation have been reported [21] to be $1.5,1.5,0.4,0.08$ and 0.1 $\mathrm{cm}^{-1}$ respectively. The E4 and E5 peaks are not completely resolved from each other in the asirradiated material. Their apparent ionization activation energies are $0.72 \pm 0.03 \mathrm{eV}$ and $0.88 \pm$ $0.08 \mathrm{eV}$ respectively. It was recently shown that recombination annealing during current injection in a p-n junction diode can significantly reduce the E5 DLTS signal amplitude without affecting that of E4 [22]. This allows the separate observation of the $E 4$ DLTS peak which proved to be much wider than is expected for an isolated defect level. Moreover, we have studied E5 with the help of the Differential DLTS (DDLTS) technique [23] in order to determine its electric-field sensitivity. The E5 DDLTS peaks were found to be extremely sensitive to electric fields as low as $100 \mathrm{kV} / \mathrm{cm}$. Neither a PooleFrenkel effect nor a multiphonon-assisted field ionization of an isolated defect with a near mid-gap level can account for this abnormal electric field sensitivity of E5.

The above observations rule out the attribution of E4 or E5 to isolated point defects. Instead, we are led to attribute each of E4 and E5 to a distant defect pair, one defect having a near mid-gap level while the other has a level nearer to the conduction band. For such a pair and at low temperatures, the emission rate of a single captured electron will be very slow and a second electron can be captured and has to occupy the shallower level (by virtue of Pauli's exclusion principle). The trapping and subsequent ionization of this second electron should thus be seen as a low-temperature DLTS peak corresponding to the shallower level. Thus, the shallower level component in these defect pairs should be directly observable by DLTS and can only be E2 or E3. The low-field ionization kinetics of E2 and E3 are exponential as evidenced by their narrow DLTS peaks [21]. This indicates that the mid-gap level component of the E4 and E5 pairs should be a single donor ; otherwise, when occupied by an electron, its Coulomb potential could introduce an observable random fluctuation in the ionization energies of E2 and E3.

We are thus led to postulate the occurrence of a Mid-Gap level defect which we represent by the symbol MG in this article. Independent evidence for such an irradiation-induced mid-gap level comes in fact from EPR, Hall effect and hopping conductivity measurements as discussed below.

2.1.2 Irradiation-induced mid-gap levels in GaAs. An Electron Paramagnetic Resonance (EPR) quadruplet structure attributed to the antisite $\mathrm{As}_{\mathrm{Ga}}^{+}$can be obtained in concentrations up to $10^{18} \mathrm{~cm}^{-3}$ by electron irradiation $[24,25]$. Similar EPR spectra are observed after plastic deformation or fast neutron irradiation [3] of n- or p-GaAs. The photo-EPR response for plastically deformed materials shows that the EPR signal is related to a mid-gap single donor level. Electrical conductivity and Hall effect measurements show that the Fermi level is pinned near mid-gap after large doses of either fast electron [4] or fast neutron [6] irradiation. These observations suggest that the $\mathrm{As}_{\mathrm{Ga}}$ defect is associated with the mid-gap single donor level MG that was postulated above for the interpretation of DLTS data.

The famous EL2 single donor, which is native in bulk and vapour phase epitaxial materials, is probably a complex including the $\mathrm{As}_{\mathrm{Ga}}$ defect [1-6]. Identifying together the two $\mathrm{As}_{\mathrm{Ga}}$ related mid-gap single donors MG and EL2 is still a matter of discussion. Our observations suggest that E4 and E5 are due to defect pairs each consisting of the $\mathrm{As}_{\mathrm{Ga}}$ related mid-gap donor together with one of the acceptors E2 or E3, which are both believed to be associated with a $V_{\text {As }}$ vacancy [21].

We shall demonstrate in a later section that largedose irradiation gives rise to a nearest neighbour hopping DC conductivity through a mid-gap defect with practically the same lattice relaxation parameters $\hbar \omega$ and $S \hbar \omega$ as those of EL2.

2.1.3 Defects in heavy-particle bombarded GaAs. Detailed DLTS data have been published [26] for boron ion implanted n-GaAs. The DLTS spectra observed, as can be seen seen in figure 1, consist of a wide high temperature peak, referred to as the Upeak, together with shallower levels. Experiments [27] show that the main observable shallow level in the implanted material is level E3 $\left(E_{\mathrm{c}}-\right.$ $0.32 \mathrm{eV}$ at $0 \mathrm{~K}$ ). Both the observed peak position 


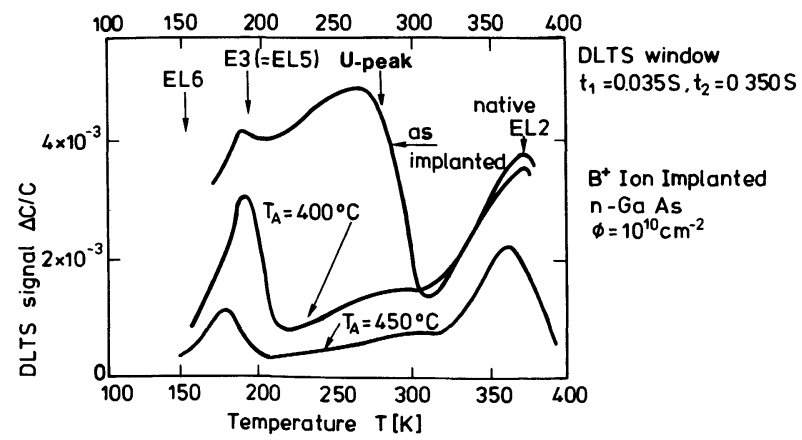

Fig. 1. - DLTS spectra for $100 \mathrm{keV}$ boron-ion implanted $\mathrm{n}$-GaAs after $30 \mathrm{~min}$ isochronal annealing at different temperatures, from reference [26]. In the starting material, the free electron concentration was about $10^{17} \mathrm{~cm}^{-3}$. Gold Schottky diodes were used. The reverse bias voltage and the filling bias pulse amplitude were both $2 \mathrm{~V}$.

and its high electric field sensitivity [28] confirm that this defect is identical with defect level E3 generated in fast electron proton bombarded materials [17]. Other authors [16] also report a small DLTS signal related to level $\mathrm{E} 2\left(E_{\mathrm{c}}-0.14 \mathrm{eV}\right)$ in ion-implanted materials which is again identified with that observed in electron-irradiated materials.

As to the high temperature U-peak, we shall confirm in this article its attribution [26] to electronic interactions between the mid-gap defect level MG with the shallower defect E2 and E3.

The large defect density at the clusters' core solidly pins the Fermi level at mid-gap and, as will be discussed in section 6.2, only defects at the clusters outskirts can have an observable influence on DC electrical conductivity and DLTS results. The damage energy imparted per lattice atom at these outskirts is expected to be of the same order as that imparted during fast-electron irradiation and the same type of individual point defects should be created. Moreover, it will be shown in section 6 that the shallower defects can only be observed by DLTS when they are well away from the cluster cores. One therefore understands why the same isolated shallow traps (E2, E3) as in electron irradiated materials are observed. In the next sections we shall see that the experimental results for heavy-particle bombarded semi-insulating and $\mathrm{n}-\mathrm{GaAs}$ can be explained by invoking the same defects (E2, E3, MG) as for fastelectron-irradiated materials with the same relative concentrations.

2.2 DATA USED IN OUR CALCULATIONS. - In our calculations we shall make use of the properties known for the above defects and listed in table I. An important input to our calculations is the donor or acceptor nature of the defects involved. This is because Coulomb potentials introduce an important term in the difference between interacting defect levels (see Eq. (5)).
The defect related electron effective masses $m_{\mathrm{d} i}$ entering equation (2) are taken, in all cases, to be 0.25 in units of the free-electron mass. This value together with reasonable assumptions for the defect potential near the centroid (for $\left|\mathbf{r}-\mathbf{r}_{i}\right|<a_{i}$ ) lead, within our quantum defect model, to ionization energies comparable to those of table I. The numerical value of the square of the wave function preexponential factor $\boldsymbol{\eta}_{i}^{2}$ is given in table $I$ as deduced from the electric-field ionization data (in Tab. I of Ref. [10] this factor is represented by the symbol $3 \hbar \gamma / 2)$. We can thus evaluate the product of the pre-exponential factors in the expression of $P(E)$ appearing in equation (2) within an uncertainty of about a factor of 3 .

Apart from as-irradiated defects, we have also listed EL6 which is native in bulk GaAs and is probably identical to a defect P1 observed (in low concentrations) after fast-electron irradiation and annealing [32]. This defect is worth studying because it has an electron ionization activation energy almost identical with that of E3 and can be confused with it in DLTS experiments. EL6 can be differentiated from E3 because of the very different behaviour of their DDLTS peak position at different electric fields. E3 is known to be very sensitive to the electric field [28] whereas EL6 is practically insensitive to the field [27]. This difference in the electric-field behaviour of E3 and EL6 can be accounted for, according to the theory of reference [6], by the abnormally large lattice relaxation energy of EL6 $[27,33](S \hbar \omega \simeq 600 \mathrm{meV})$.

In the following sections we shall refer, in our interpretations, mainly to the defects MG, E2 and E3. Experimental observations in irradiated GaAs will be shown to be a result of electronic interactions between these defects. As for E1, we recall that it is a second charge state of E2. In our notation, we shall reserve label $i=1$ for the mid-gap defect MG.

In the heavily damaged regions of irradiated GaAs the Fermi level is, as already discussed, pinned on the mid-gap level MG. If $f_{0}$ is the average thermal equilibrium occupation ratio of this MG single donor level, the fraction $\left(1-f_{0}\right)$ of these defects will be positively charged and the rest will be neutral. Since other defect levels, at all temperatures of interest, are many $k T$ units above or below the Fermi energy, they can only introduce a temperature-independent charge density. Overall charge neutrality, therefore, implies a temperatureindependent value of $f_{0}$.

\section{DC hopping conductivity in irradiated GaAs.}

Deng et al. [34] have extensively studied the DC electrical conductivity induced in GaAs by heavyparticle $\left(\mathrm{Ar}^{+}\right)$bombardment, which causes damage in a layer approximately $0.4 \mu \mathrm{m}$ thick. Their conduc- 
Table I. - Physical parameters characteristic of the individual $\mathrm{n}-\mathrm{GaAs}$ deep-level defects as assumed in the numerical evaluations. Our calculations proved tolerant to the indicated uncertainties in phonon energies $\hbar \omega$ and Franck-Condon shifts Sh $\omega$. The dimensionless constant $\eta_{i}^{2}$ is the square of the pre-exponential factor appearing in equation (1) for the trapped electron wave-function. It is deduced by comparing the experimental electric-field ionization with the predictions of the theory of reference [10]. The largest parameter uncertainty is that of $\eta_{i}^{2}$ and is related to experimental uncertainties in the electric fields. The ionization energy is assumed to depend on temperature according to a law similar to that of the GaAs band-gap (Ref. [29]), i.e.

$$
\Delta_{T}(T)=\Delta_{T}(0)-\gamma T^{2} /(T+200)
$$

where the temperature coefficient $\gamma$ is determined from the deep level electron capture and emission data (Ref. [30]). E1 has a large lattice relaxation. Its negative (repulsive) potential suggested by the small capture cross-section ( $\simeq 10^{-18} \mathrm{~cm}^{-2}$ reported in Ref. [31]) is confirmed by the small experimentally determined value of $\eta_{i}^{2}$. E2 has a large capture cross-section $\left((1.3 \pm 0.3) \times 10^{-13} \mathrm{~cm}^{-2}\right.$ as shown in Ref. [31]). Since $\mathrm{E} 1=\mathrm{E} 2+$ electron, E1 and E2 can only be double and single acceptor levels respectively. The data for EL6 are incomplete. Because of its very large Franck-Condon Shift, we have assumed that its electron capture crosssection has a small Arrhenius activation energy.

\begin{tabular}{|c|c|c|c|c|c|c|}
\hline $\begin{array}{c}\text { Defect } \\
\text { label } \\
\text { and type }\end{array}$ & $\begin{array}{c}\Delta_{T} \\
\mathrm{meV}\end{array}$ & $\begin{array}{c}\gamma \\
\mathrm{meV} / \mathrm{K}\end{array}$ & $\begin{array}{c}\hbar \omega \\
\mathrm{meV}\end{array}$ & $\begin{array}{l}\text { Sh } \omega \\
\mathrm{meV}\end{array}$ & $\begin{array}{c}\eta_{i}^{2} \\
\text { dimensionless }\end{array}$ & $\begin{array}{l}\text { References } \\
\text { for data }\end{array}$ \\
\hline $\begin{array}{l}\text { E1 } \\
\text { Double } \\
\text { Acceptor }\end{array}$ & 45 & 1 & $4 \pm 2$ & $60 \pm 20$ & $(1.1 \pm 0.9) \times 0.0001$ & $\begin{array}{l}\text { This work using } \\
\text { theory of Ref. [10] } \\
\text { to fit experiments } \\
\text { of Ref. [31] } \\
\text { Confirmed by } \\
\text { the temperature } \\
\text { dependence of } \\
\text { the electron } \\
\text { capture rates }\end{array}$ \\
\hline $\begin{array}{l}\text { E2 } \\
\text { Single } \\
\text { Acceptor }\end{array}$ & 140 & 0 & $4 \pm 1$ & $55 \pm 5$ & $0.02 \pm 0.015$ & $\begin{array}{l}\text { This work using } \\
\text { theory of Ref. [10] } \\
\text { to fit experiments } \\
\text { of Ref. [31] }\end{array}$ \\
\hline $\begin{array}{l}\text { E3 } \\
\text { Single } \\
\text { Acceptor }\end{array}$ & 310 & 0.17 & 10 & $100 \pm 20$ & $0.02 \pm 0.005$ & Refs. $[10,11,28]$ \\
\hline $\begin{array}{l}\text { EL6 = P1 } \\
\text { Probably } \\
\text { Single } \\
\text { Acceptor }\end{array}$ & $320 \pm 30$ & $0.2 \pm 0.1$ & $\begin{array}{c}\text { unknown, } \\
\text { but assuming } \\
\hbar \omega<k T\end{array}$ & $600 \pm 100$ & taken 0.02 & Refs. [27, 33] \\
\hline $\begin{array}{l}\text { MG or EL2 } \\
\text { Single } \\
\text { Donor }\end{array}$ & 735 & 0.27 & $20 \pm 2$ & $145 \pm 10$ & $0.08 \pm 0.03$ & $\begin{array}{l}\text { Refs. }[10,11] \text {. Also } \\
\text { using theory } \\
\text { of section } 4 \text { of } \\
\text { this work to fit } \\
\text { experiments } \\
\text { of Ref. [34]. }\end{array}$ \\
\hline
\end{tabular}

tivity increased with ion dose but saturated at about a dose of $3 \times 10^{13} \mathrm{Ar}^{+} \mathrm{cm}^{-2}$. Both the type of temperature dependence observed and the magnitude of thermo-power allow the attribution of this conductivity to electron hopping. However, Deng et al. accounted for their experimental results with a variable range hopping model [35] and they had to assume a continuous defect density energy distri- 
bution in the band-gap. This defect distribution is in clear conflict with DLTS and EPR observations. Furthermore, an essential condition for the occurrence of multiple range hopping is that first and second neighbour hopping probabilities should be comparable [35]. This, in fact, implies complete amorphization for large-dose heavy-particle bombardment, in contradiction with the X-ray diffraction data of Coates and Mitchell [36]. For the ion bombardment doses needed to approach the saturation hopping DC conductivity, the defect concentration can be roughly estimated by extrapolating DLTS data [26] to be of the order of $10^{19} \mathrm{~cm}^{-3}$. An estimate is also obtained from EPR of fast-neutronirradiated materials [6], in which case the onset of hopping conduction is observed when the $\mathrm{As}_{\mathrm{Ga}}^{+}$ defects are in a concentration of $10^{18} \mathrm{~cm}^{-3}$ [7]. For these concentrations, nearest neighbour hopping probabilities are expected to exceed those for second neighbour hopping by several orders of magnitude.

We have calculated the nearest neighbour hopping conductivity according to the theory of reference [9]. Our DC conductivity calculations are an extension of the Miller and Abrahams theory [37] for percolation conduction but include in addition multiphonon effects. The expression we use for DC hopping conductivity $\sigma_{\mathrm{dc}}$ between mid-gap level defects (labelled 1) can be written as

$$
\sigma_{\mathrm{dc}}=\frac{3 q^{2} B_{\mathrm{c}}^{2}}{8 \pi r_{11}^{2} \kappa} \cdot \frac{f_{0}\left(1-f_{0}\right)}{k T} \cdot \mathrm{e}^{-\varepsilon_{\mathrm{c}} / k T} \cdot R_{11}\left(r_{11}\right)
$$

where $\kappa=\frac{1}{2} \cdot \mathrm{d}\left(\ln \left(R_{11}\right)\right) / \mathrm{d} r_{11} . \quad B_{\mathrm{c}}$ stands for the average number of above critical conductances emerging from each site at percolation threshold and can be taken to be 2.7 in accordance to Monte Carlo simulations for three-dimensional uniform random distribution of defects $[38,39]$. The critical interdefect distance $r_{11}$ is related to the average mid-gap defect (MG) concentration $n_{\mathrm{MG}}$ by $\frac{4 \pi}{3} r_{11}^{3} n_{\mathrm{MG}}=$ $B_{\text {c. }}$.

The hopping rate $R_{11}\left(r_{11}\right)$ appearing in the above expression is calculated for zero electric fields (same initial and final energies) and for MG defects separated by a distance $r_{11}$. The activation energy $\varepsilon_{\mathrm{c}}$ corresponds to random fluctuations of the Coulomb potential in accordance to the theory of Miller and Abrahams [37]. Introducing this activation energy very considerably improves the agreement with experimental results as compared to our earlier calculations where we did not take it into account [40].

Figure 2 shows best fits of our theoretical results for $\sigma_{\mathrm{dc}}$ with the experimental results of Deng et al. for as-implanted samples. The high-temperature activation energy can be proven to be equal to

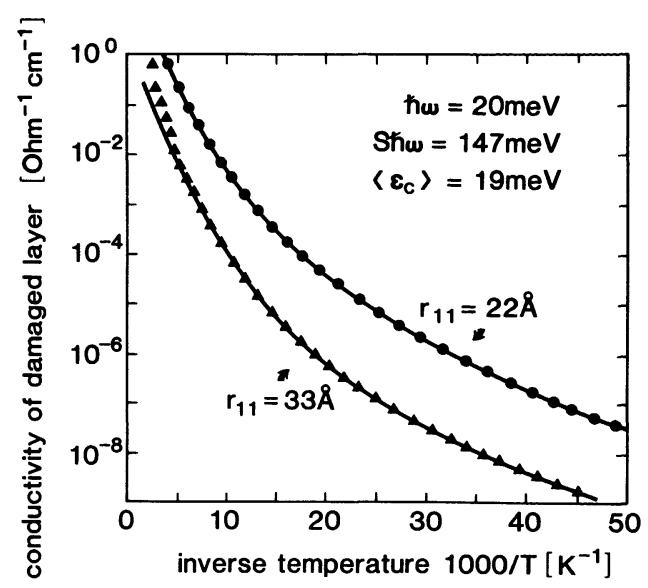

Fig. 2. - Comparison of theory and experiment for the low-temperature electrical conductivity. The experimental points are from Deng et al. in reference [34] for large dose Ar bombarded GaAs. The full circles correspond to asbombarded samples while the full triangles correspond to a $400{ }^{\circ} \mathrm{C}$ annealed sample. The curves are calculated for hopping between MG defects. For both curves we have taken $f_{0}=1 / 2$.

$\varepsilon_{\mathrm{c}}+(S \hbar \omega-3 k T) / 2$ so that the Franck-Condon shift $S \hbar \omega$ of MG is directly deduced. The value of the activation energy $\varepsilon_{\mathrm{c}}$ is deduced from the behaviour of $\sigma_{\mathrm{dc}}$ in the low temperature limit $(T<$ $50 \mathrm{~K}$ ) where it is responsible for the observed activation energy. The phonon energy $\hbar \omega$ is deduced from the plot of $\log \left(\sigma_{\mathrm{dc}}\right)$ versus $1 / T$ for intermediary temperatures $(200>T>100 \mathrm{~K})$. It is remarked that the fit gives very good agreement with experimental results with a slight discrepancy only at the highest temperatures in the $400{ }^{\circ} \mathrm{C}$ annealed samples. This discrepancy can be completely attributed to hopping conduction through levels shallower than MG [9]. This shallow levels contribution is expected to be significant at high temperatures and when the defect densities are relatively low (as is the case after thermal annealing).

The phonon best-fit parameters deduced in the above manner are $\hbar \omega=20 \pm 2 \mathrm{meV}$ and $S \hbar \omega=$ $145 \pm 10 \mathrm{meV}$, which are in excellent agreement with those reported $[10,11]$ for the native mid-gap level EL2 $(\hbar \omega=20 \pm 5 \mathrm{meV}$ and $S \hbar \omega=140 \pm$ $10 \mathrm{meV})$.

Miller and Abrahams [37] have tabulated the normalized theoretical values of the activation energy $\varepsilon_{\mathrm{c}}$ (in their article they used the symbol $\varepsilon_{3}$ in place of $\varepsilon_{\mathrm{c}}$ ) for different compensation ratios. Our best-fit procedure yields $\varepsilon_{\mathrm{c}}=19 \pm 2 \mathrm{meV}$ which agrees with the predictions of Miller and Abrahams theory for the mid-gap defect concentrations deduced from figure 2 provided we take a compensation ratio of $\left(1-f_{0}\right)=0.5 \pm 0.2$ for the as irradiated sample and $\left(1-f_{0}\right) \simeq 0.1$ for the annealed sample. We therefore see that the different 
parameters needed for the fit are self-consistent. However, the above estimates of the compensation ratio $\left(1-f_{0}\right)$ must be considered as rough because of the uncertainty in the estimated inter-defect separations ( $r_{11}$ values in Fig. 2).

\section{AC hopping conductivity in GaAs.}

A non-zero DC electron hopping conductivity $\sigma_{\mathrm{dc}}$ implies the availability of uninterrupted hopping paths between the electrodes. Another type of hopping-related conductivity can be observed and does not imply the occurrence of such continuous conduction paths. In the presence of an AC electric field, dielectric polarization losses [41] give rise to an additional AC conductivity component $\sigma_{\mathrm{ac}}$, and thus the total electrical conductivity $\sigma_{\text {tot }}$ should be expressed as a sum $\sigma_{\mathrm{tot}}=\sigma_{\mathrm{dc}}+\sigma_{\mathrm{ac}}$. The AC electric field induces dielectric dipoles; a lag angle in the time-response of these induced dipoles with the field gives rise to power losses which can be expressed as a conductivity $\sigma_{\mathrm{ac}}$.

Pollak and Geballe [42] made the corresponding calculations exclusively taking into account pairs of centres. This isolated defect pairs approximation is shown to be valid only for angular frequencies $\omega_{\mathrm{s}}$ higher than the hopping rate $R_{12}\left(r_{0}\right)$ corresponding to the average separation $r_{0}$ between the centres [42]. The direct Kirchoff's law numerical simulations of McInnes et al. [43] have later shown that the isolated defect pairs approximation gives indeed a good estimate of $\sigma_{\text {ac }}$ for high frequencies $\left(\omega_{\mathrm{s}}>\right.$ $R_{12}\left(r_{0}\right)$ ). At lower frequencies, this approximation fails but this is of little consequence since the AC conductivity has always to be added up to the DC conductivity and this swamps any erroneous evaluation. Making this simplifying isolated pairs assumption, the AC loss-conductivity $\sigma_{\text {ac }}$ can be obtained by adding up the contributions of the defects having separations between $r_{12}=r$ and $r_{12}=r+\mathrm{d} r$ from $r=0$ to $\infty$, thus

$$
\begin{aligned}
& \sigma_{\mathrm{ac}}=N_{\mathrm{p}} \int_{r=0}^{\infty}\left(f_{1}\left(1-f_{2}\right)+f_{2}\left(1-f_{1}\right)\right) \times \\
& \times \frac{(q r)^{2} \cdot \exp \left(\frac{E_{T 2}-E_{T 1}}{k T}\right)}{3 k T\left(1+\exp \left(\frac{E_{T 2}-E_{T 1}}{k T}\right)\right)^{2}} \\
& \times \frac{\omega_{\mathrm{s}}^{2} \cdot R_{\mathrm{ss}}}{\omega_{\mathrm{s}}^{2}+R_{\mathrm{ss}}^{2}} \cdot \frac{\mathrm{d} P(r)}{\mathrm{d} r} \cdot \mathrm{d} r
\end{aligned}
$$

where $R_{\mathrm{ss}}=R_{12}+R_{21}$ and $N_{\mathrm{p}}$ is the volume concentration of nearest-neighbour pairs. Only those pairs with one defect occupied with an electron and the other empty are effective. This accounts for the factor $\left(f_{1}\left(1-f_{2}\right)+f_{2}\left(1-f_{1}\right)\right)$ appearing in the above expression; $f_{1}$ and $f_{2}$ being the steady-state
Fermi-Dirac occupation probabilities of levels 1 and 2 respectively. $E_{T 1}$ and $E_{T 2}$ are the total energies of the two centres involved. $P(r)$ is the portion of these pairs separated by distances smaller than $r$.

From equation (7), the defect levels most likely to contribute to the above $\mathrm{AC}$ conductivity prove to be those situated very near the Fermi level. For undoped semi-insulating or heavy dose irradiated GaAs, these defects can only be EL2 or MG respectively. In both cases, we may take for $N_{\mathrm{p}}$ half the total defect concentration $N_{T}$, and for $P(r)$ we take a Poisson distribution given by [44]

$$
P(r)=1-\exp \left(-\left(\frac{r}{r_{0}}\right)^{3}\right)
$$

$$
\text { where } \quad r_{0}=\left(\frac{3}{4 \pi N_{T}}\right)^{1 / 3} \text {. }
$$

We have numerically evaluated the above integral expression for $\sigma_{\mathrm{ac}}$. Our numerical results differ from those earlier published for shallow impurity-band AC conduction [42, 43] since our transfer rates include multi-phonon hopping effects.

Comparison with experimental results. - Figure 3 reports the loss part of the defect-related permittivity defined by $\varepsilon^{\prime \prime} \equiv \frac{\sigma_{\mathrm{ac}}+\sigma_{\mathrm{dc}}}{\omega_{\mathrm{s}}}$, calculated as a function of the frequency $\frac{\omega_{\mathrm{s}}}{2 \pi}$ for different defect densities $N_{T}$ corresponding to different average defect separations $r_{0}$. The results are normalized to $\varepsilon_{\infty}$, which is the intrinsic dielectric permittivity of the medium in the absence of defects. In the calculations, we

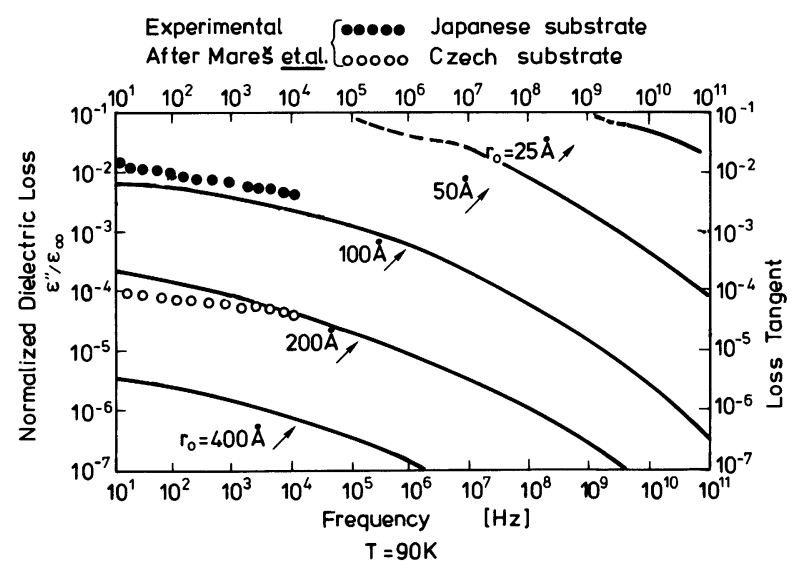

Fig. 3. $-\varepsilon^{\prime \prime} / \varepsilon_{\infty}$ (practically equal to the dielectric loss tangent) at $T=90 \mathrm{~K}$ as a function of frequency for different average defect separations $r_{0}$. The dashed parts of the curves for $r_{0}=25$ and $50 \AA$ correspond to a dominant influence of the DC conductivity. The experimental points are from Mareš et al. in reference [45] for two high purity semi-insulating GaAs substrates. The results correspond to true homogeneous bulk dielectric losses (guard rings used and surface not heavily damaged). 
assumed EL2 defects and we took for the compensation ratios $\left(1-f_{1}\right)=\left(1-f_{2}\right)=1 / 2$.

Mareš et al. [45] have performed dielectric loss measurements in the frequency range from $10 \mathrm{~Hz}$ to $10 \mathrm{kHz}$ for undoped semi-insulating GaAs. These authors showed that the experimental measurements are very sensitive to surface treatments and they showed that it is essential to use guard rings in order to obtain reliable results. For undoped GaAs, the EL2 native mid-gap defect concentration is a few $10^{16} \mathrm{~cm}^{-3}$, thus corresponding to an average defect separation in the range of 150 to $250 \AA$. Our predictions (curves in Fig. 3) agree both in order of magnitude and in frequency dependence with the experimental results (empty and full circles in Fig. 3) of these authors.

The above calculations and experimental results refer to a temperature of $90 \mathrm{~K}$. For the above mentioned EL2 concentrations and frequencies, our model predicts only slight changes in the AC dielectric losses when the temperature changes from 90 to $300 \mathrm{~K}$, in agreement with the experimental observations of Mareš et al. For larger defect densities $\left(r_{0}<50 \AA\right)$, the low-frequency $90 \mathrm{~K}$ dielectric loss is predicted to be mainly due to the DC conductivity $\sigma_{\mathrm{dc}}$ (dashed part of the curves for $r_{0}=25$ and $50 \AA$ in Fig. 3). The corresponding $\varepsilon^{\prime \prime}$ tends to $\varepsilon^{\prime \prime} \simeq$ $\frac{\sigma_{\mathrm{dc}}}{\omega_{\mathrm{s}}}$ which is inversely proportional to frequency and varies with temperature proportionally to $\sigma_{\mathrm{dc}}$ as was studied in the previous section.

For microwave frequencies ( 4 to $40 \mathrm{GHz}$ ) the dielectric loss tangent (nearly equal to $\varepsilon^{\prime \prime} / \varepsilon_{\infty}$ ) has been measured at and above room temperature for semi-insulating GaAs [46] and tends to increase with frequency. This behaviour cannot be reproduced by a model with a uniform random distribution of defects. Instead, one is led to suggest the occurence of zones with highly localized defect concentrations. In these zones the DC conductivity can be locally high. The dielectric function of a semiconductor with such inclusions can be predicted from a standard formula for a mixture [45]. Only a very small fraction (of the order of $10^{-4}$ ) of the semiconductor's volume with a high - but non-metallic - DC conductivity can account for the dielectric loss at microwave frequencies. Such microscopic high $\sigma_{\mathrm{dc}}$ regions may occur, for example, within a disturbed layer near the semiconductor's surface. The role of such a near-surface layer is confirmed by the observations of Mareš et al. who report a marked increase in $\varepsilon^{\prime \prime}$ which becomes nearly proportional to frequency (in the range of a few $\mathrm{kHz}$ ) after a surface oxidation of semi-insulating GaAs.

\section{Electron ionization rates of defect pairs.}

We now predict the transient ionization behaviour of a defect pair consisting of a deep level defect (labelled 1) and a shallower level (labelled 2). Assuming that a single electron is trapped in the defect pair, it will occupy level 2 for a fraction $f_{2}$ of the trapping time. During the remaining fraction $\left(1-f_{2}\right)$ of this time, the electron will occupy level 1 . The value of $f_{2}$ can be determined from the quasi-equilibrium condition

$$
\begin{array}{cc}
f_{2}\left(e_{2}+R_{21}\right)=\left(1-f_{2}\right) R_{12} \\
\text { and thus } \quad f_{2}=\frac{R_{12}}{R_{21}+R_{12}+e_{2}},
\end{array}
$$

where $e_{2}$ is the electron emission rate from level 2 . The trapped electron can either be directly ionized from the ground level (1) at a rate $e_{1}$ or, indirectly through level (2), at a rate $e_{\text {ind }}$ given by

$$
e_{\text {ind }}=f_{2} e_{2}=\frac{R_{12} e_{2}}{R_{21}+R_{12}+e_{2}} .
$$

It must be pointed out that each of the direct ionization rates $e_{1}$ and $e_{2}$ from defects 1 and 2 differs from that of an isolated defect since the Coulomb potential of the neighbouring defect changes its ionization energy. Taking this potential term into account amounts to changing the ionization energies of defects 1 and 2 from $\Delta_{T 1}$ and $\Delta_{T 2}$ to $\Delta_{T 1}+\frac{\alpha_{2}}{r_{12}}$ and $\Delta_{T 2}+\frac{\alpha_{1}}{r_{12}}$ respectively. We deduce the emission rate $e_{i}$ from $e_{i 0}$ of the isolated defect from the relationship

$$
e_{i}=e_{i 0} \frac{D_{i}\left(\Delta_{T i}+\frac{\alpha_{j}}{r_{12}}\right)}{D_{i}\left(\Delta_{T i}\right)}
$$

where $i \neq j$, and it is recalled that $D_{i}\left(E_{\mathrm{p}}\right)$ represents the probability that an electron trapped in defect $i$ will absorb a multiphonon energy $E_{\mathrm{p}}$ (Eq. (3)).

In the above treatment, the indirect ionization rate $e_{\text {ind }}$ was written as $f_{2} e_{2}$ which is a « quasi-static occupation ratio" approximation. A more exact treatment by Rees et al. [47] gives practically identical results provided the energy difference between the two levels is large enough to guarantee the inequality $R_{12} \ll R_{21}$. This condition is largely met for the defect pairs we are dealing with. Adding up direct and indirect components, we obtain for the total electron ionization rate $e_{n}$ :

$$
e_{n}=e_{1}+\frac{R_{12} e_{2}}{R_{21}+e_{2}} .
$$

The above emission rate $e_{n}$ is a function of the electric field since the hopping rates $R_{12}$ and $R_{21}$ as well as the individual defect ionization rates $e_{1}$ and $e_{2}$ are all field-dependent. The field dependence of the ionization rates of all the levels involved 
in our present study is known from previous work $[10,26,27,31]$. The field dependence of the rates $R_{12}$ and $R_{21}$ comes mainly from the contribution $q \cdot F \cdot r_{12} \cos (\theta)$ to the difference in the energy levels (see Eq. (5)). Two extreme cases can be recognized with equation (8) :

i) If the inequality $R_{21} \gg e_{2}$ holds (level 2 not too shallow), equations (4), (8) yield

$$
e_{n}=e_{1}+\exp \left(\frac{E_{T 1}-E_{T 2}}{k T}\right) \cdot e_{2} .
$$

ii) If the reverse inequality $R_{21} \ll e_{2}$ holds (level 2 is shallow), we have

$$
e_{n}=e_{1}+R_{12}
$$

and the indirect electron emission rate is governed by the internal transition rate $R_{12}$.

The above results can be used to make computer simulations of the DLTS response of defect pairs. We use equation (8) to calculate the apparent electron-emission rate $e_{n}$ which must, in general, be treated as a function of the junction electric field $F$ and of the angle $\theta$ between the electric field and the line joining the defect centroids. The transient occupation ratio $Y(t, \theta)$ for a defect pair filled at time $t=0$ with an electron will be

$$
Y(t, \theta)=\exp \left(-e_{n}(\theta, F) \cdot t\right) .
$$

Averaging over a random distribution of $\boldsymbol{\theta}$ (equivalent to integrating over a $4 \pi$ steradians solid angle), we get

$$
\bar{Y}(t)=\frac{1}{2} \int_{\theta=-\pi}^{+\pi} \exp \left(-e_{n}(\theta, F) \cdot t\right) \cdot \sin \theta \mathrm{d} \theta,
$$

where we have assumed a constant inter-defect separation $r_{12}$. Calculating the DLTS response for a defect pair would amount to replacing the usual exponential ionization kinetics with equation (10). For the other more conventional aspects of DLTS simulation, the reader is referred to references [ 48 , 49].

\section{Electron ionization rates for densely packed de- fects.}

We extend the above treatment to a large number of densely packed defects. This case is quite distinct from that of isolated point defects or defect pairs. In our DLTS experiments, the defects are situated in the depleted region of a reverse-biased Schottky diodes fabricated on $\mathrm{n}-\mathrm{GaAs}$. The individual defects are initially filled with electrons by means of a filling bias pulse during which the diode depleted region is reduced. The defect density is usually larger than the initial electron concentration. We thus expect that only a small fraction of the defects will capture electrons during the filling pulse. Consequently, the DLTS peak amplitudes cannot be used to reliably estimate the defect concentrations. Furthermore, shallower defects with energies well above the Fermi-level will undergo very small changes in their electron occupation rates and will be more difficult to see than the defects with energies near to the Fermi-level. We therefore concentrate in the following treatment on the indirect emission through shallower levels of electrons previously trapped in the defect nearest to the Fermi-level.

Densely packed defects can occur in many situations. For example, a large dose of fast-electron, fast-neutron or fast-ion irradiation would lead to a high density of uniformily and randomly distributed defects. Another case of interest is that of defect grouped in clusters as could be generated by a moderate dose of heavy-particle bombardment. In the following, we first deal with the extreme case of uniformily distributed defects and we then deal with the opposite extreme case of defects grouped in well defined clusters.

6.1 UNIFORMILY DISTRIBUTED DENSELY PACKED DEFECTS. - To simplify the notation, we assume the occurrence of three electron trap types $(1,2$ and 3) with label 1 referring to the mid-gap defect (MG). In fact, the generalization to any number of defects with energy levels in the band-gap is straightforward. We can see from section 3 that the transition rates between defects of the same energy level can be extremely large (much larger than any of the ionization rates) even for separations as large as $100 \AA$ corresponding to concentrations around $2.5 \times$ $10^{17} \mathrm{~cm}^{-3}$. One can therefore treat the three levels ( $i=1,2$ and 3 ) as three bands with $N_{1}, N_{2}$, $N_{3}$ available electronic states respectively.

To calculate the ionization kinetics of the combination, we generalize the treatment of section 5 in order to determine the quasistatic electron occupation probabilities $f_{2}$ and $f_{3}$ of levels 2 and 3 in terms of $f_{1}$ of the deepest level. For any permutation $i, j, k$ of the three labels $1,2,3$, one should have :

$$
\begin{aligned}
N_{i} N_{j}\left[f_{i}\left(1-f_{j}\right) R_{i j}-f_{j}\left(1-f_{i}\right)\right. & \left.R_{j i}\right]+ \\
+N_{k} N_{j}\left[f_{k}\left(1-f_{j}\right) R_{k j}\right. & \left.-f_{j}\left(1-f_{k}\right) R_{j k}\right]- \\
& -N_{j} f_{j} e_{j}=0
\end{aligned}
$$

where the symbols $e_{j}$ represent as before the emission rates from the different individual defects. $R_{i j}$ stands, in general, for the average electron tunnelling rate between defect levels $i$ and $j$. For densely packed defects, the term $N_{j} f_{j} e_{j}$ can be neglected compared to the others in equation (11). Within this approximation, and making use of the detailed balance equation (Eq. (4)), we obtain for $i=2$ or $i=3$ 


$$
\begin{aligned}
f_{i}=\frac{1}{1+\frac{1-f_{1}}{f_{1}} \exp \left(\frac{E_{T i}-E_{T 1}}{k T}\right)} & \\
& \simeq \frac{f_{1}}{1-f_{1}} \exp \left(\frac{E_{T 1}-E_{T i}}{k T}\right)
\end{aligned}
$$

which is thus seen to follow a Fermi-Dirac distribution. More general solutions to equation (11) can be obtained for cases where, in contrast to the above treatment, the terms $N_{j} f_{j} e_{j}$ cannot be neglected. Since one expects $f_{2} \ll 1$ and $f_{3} \ll 1$, equation (11) for $j=2$ and $j=3$ yields simply a set of two linear equations in two unknowns. When this is done, we obtain results practically identical with equation (12) provided the following inequalities hold :

$$
e_{2} \ll N_{1} R_{21}\left(1-f_{1}\right)+N_{3} R_{23}
$$

and

$$
e_{3} \ll N_{1} R_{31}\left(1-f_{1}\right)+N_{2} R_{32} \text {. }
$$

Having determined $\frac{f_{2}}{f_{1}}$ and $\frac{f_{3}}{f_{1}}$, the ionization kinetics of the closely packed defects can be predicted from

$$
N_{1}\left(\frac{\mathrm{d} f_{1}}{\mathrm{~d} t}\right)=N_{1} f_{1} e_{1}+N_{2} f_{2} e_{2}+N_{3} f_{3} e_{3}
$$

Thus, provided inequalities (13) hold, the corresponding effective electron emission rate can be written as

$$
\begin{aligned}
e_{n}=e_{1}+\frac{N_{2}}{N_{1}^{*}} \exp ( & \left.\frac{E_{T 1}-E_{T 2}}{k T}\right) e_{2}+ \\
& +\frac{N_{3}}{N_{1}^{*}} \exp \left(\frac{E_{T 1}-E_{T 3}}{k T}\right) e_{3}
\end{aligned}
$$

where

$$
N_{1}^{*}=\left(1-f_{1}\right) N_{1} \text {. }
$$

As can be seen from equation (14), the emission kinetics of the defect collection cannot, strictly speaking, be described by a linear differential equation because of the occurrence of the term $\left(1-f_{1}\right)$. We can, however, approximate $\left(1-f_{1}\right)$ to a constant because the quasi-Fermi level of the closely packed defects is expected to change only slightly during the ionization transient.

It should be noted that the electron emission rate of equation (14) is sensitive to the defect concentration ratio $\frac{N_{i}}{N_{1}}$. This ratio represents the number of different paths through which an electron trapped in the deepest level 1 can be ionized via level $i$. We also note the sensitivity of the emission rate to $\left(1-f_{1}\right)$ and thus to the compensation ratio or quasi-Fermi level of the mid-gap defects.
6.2 DEFECTS GROUPED IN WELL DEFINED CLUSTERS. - We now deal with the other extreme case of defect densely pack in well defined clusters. In view of the appreciable hopping conductivity, demonstrated in section 3 , the clusters can be considered as conductors. For example, in the core of the clusters generated by boron-ion bombarded in $\mathrm{n}-\mathrm{GaAs}$, the defect density can be roughly estimated [9] to be of the order of $10^{19} \mathrm{~cm}^{-3}$. From elementary electrostatic considerations, it can be seen that the space charge can only be altered at the outskirt of these clusters during the DLTS bias sequence. Any variation in the junction field will only change the electron occupation of MG defects in their first layer around the clusters provided the inequality $\frac{q f_{1}}{r_{11}^{2}}>$ $q \varepsilon_{0} \varepsilon_{r} F$ holds. For fields $F$ around $200 \mathrm{kV} \mathrm{cm}^{-1}$ and with $f_{1}=0.5$, this inequality will hold provided the MG defect separation $r_{11}$ is smaller than $60 \AA$ (i.e. for MG densities larger than $3.0 \times 10^{18} \mathrm{~cm}^{-3}$ ). From the discussion in section 2, we expect the Fermi level at the clusters' core to be strongly pinned at mid-gap by the defects themselves and we, moreover, expect average charge neutrality there. Hence a DLTS experiment can only reveal those defects situated at the clusters' periphery where their density is of the order of $10^{18} \mathrm{~cm}^{-3}$ (much smaller than at the cores). Furthermore, within or in the immediate vicinity of clusters, shallow electron trapping levels cannot be filled with electrons because of the near mid-gap position of the Fermi level. In particular, it is seen from figure 4 that shallow level defects have to be separated by a minimum distance $W$ from the clusters to be observable by DLTS.

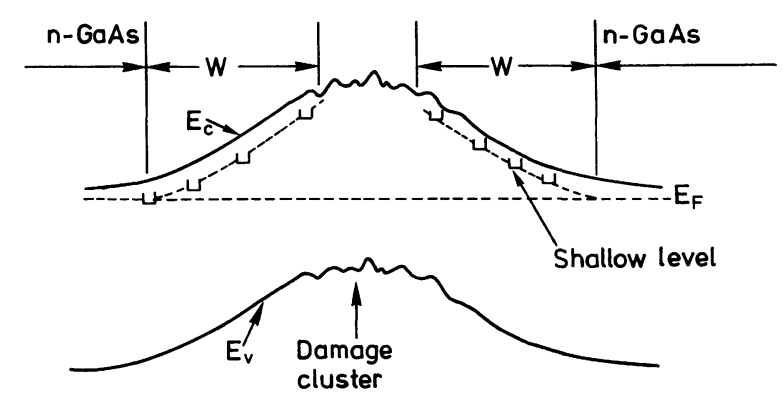

Fig. 4. - Energy band diagram across a damage cluster situated in the neutral region of heavy particle bombarded $\mathrm{n}-\mathrm{GaAs}$. The electrical properties of the defects at the cluster's core are masked because of electrostatic screening effects and because the Fermi level is strongly pinned at mid-gap there. Shallow levels at distances less than $W$ from the clusters will remain empty and will not be detected by DLTS.

We define the inside of a cluster as the region where the inter-defect hopping rates are larger than the rates observable through the DLTS rate window. 
The "well defined clusters " situation is that in which the inter-defect hopping rates decrease when one moves away from the clusters within a transition region much smaller than the cluster size. Such a situation can be assumed for our low-dose implanted samples which have not been subject to any annealing treatment. In these samples, the clusters do not overlap and the diode depleted regions are much larger than the clusters sizes. In this context and for simplicity reasons, we treat the cluster as a spherical conductor in a uniform external junction field $F$. From elementary electrostatics, one can evaluate the electric field $F_{s}(\theta)$ experienced by a defect at the cluster's periphery as a function of the angle $\theta$ between the external field and the radius-vector of the sphere. One gets

$$
F_{\mathrm{s}}(\theta)=3 F \cos (\theta) .
$$

The electron emission rate $e_{n}\left(F_{\mathrm{s}}\right)$ from a given point at the clusters outskirt can now be deduced from equation (14). The effective clusters emission rate $e_{n}^{*}$ is obtained by averaging over the surface of the spherical cluster and hence

$$
e_{n}^{*}=\frac{1}{2} \int_{\theta=-\pi}^{+\pi} e_{n}(3 F \cos (\theta)) \cdot \sin \theta \mathrm{d} \theta,
$$

\section{Interpretation of DLTS data in fast electron irradiated GaAs.}

As was discussed in section 2, one is led to attribute the E4 and E5 fast-electron irradiation DLTS peaks to the defect pairs $(M G+E 2)$ and $(M G+E 3)$. In order to verify this interpretation of E4 and E5, we have made computer simulations for the Differential DLTS (DDLTS) behaviour of the defect pairs $(M G+E 2)$ and $(M G+E 3)$. The results of section 3 allow us to take the MG phonon parameters equal to those of the EL2 native defect. Our model proved, however, to be insensitive to those MG phonon parameters. In contrast, the simulations proved extremely sensitive to variations of the phonon parameters of E2 and E3 around their experimentally known values (Tab. I). Another parameter to which our model is sensitive is, of course, the energy level of MG. Agreement with observations is obtained only if the MG level is taken within $10 \mathrm{meV}$ of that of EL2.

In figure 5, the E4 DDLTS peaks are seen to have a behaviour consistent with that predicted for (MG + E2) pairs. If the defect centroids separation $r_{12}$ is larger than $30 \AA$, the model predicts, for such pairs, an ionization rate practically equal to the internal electron hopping rate $R_{12}$ between MG and E2 (Eq. (9b). This behaviour is related to the very high electron emission rate of E2 (see Eqs. (8) and (9b) and the discussion around them). Furthermore, $R_{12}$ varies quickly with the interdefect separation $r_{12}$, but is insensitive to the electric field. Thus, the wide DDLTS peaks observed for E4 are explained by random variations of $r_{12}$ around $30 \AA$ while their insensitivity to the field is as expected for $R_{12}$. The uncertainties in the pre-exponential factors used in our model, as defined in section 2.2 , directly influence our evaluation of $R_{12}$. This leads to an uncertainty about $\pm 10 \mathrm{~K}$ in the calculated DDLTS peak positions. The agreement of the model for $r_{12} \simeq$ $30 \AA$ with the experimental results for $\mathrm{E} 4$ is well within this uncertainty. It is noteworthy that, in $(\mathrm{MG}+\mathrm{E} 2)$ pairs for which $r_{12}<30 \AA$, E2 does not behave like a shallow level defect anymore because the Coulomb potential of MG appreciably increases the effective ionization energy of E2. This explains the occurrence of a minimum in the calculated DDLTS peak temperatures of $(\mathrm{MG}+\mathrm{E} 2)$ pairs for $r_{12}=30 \AA$.

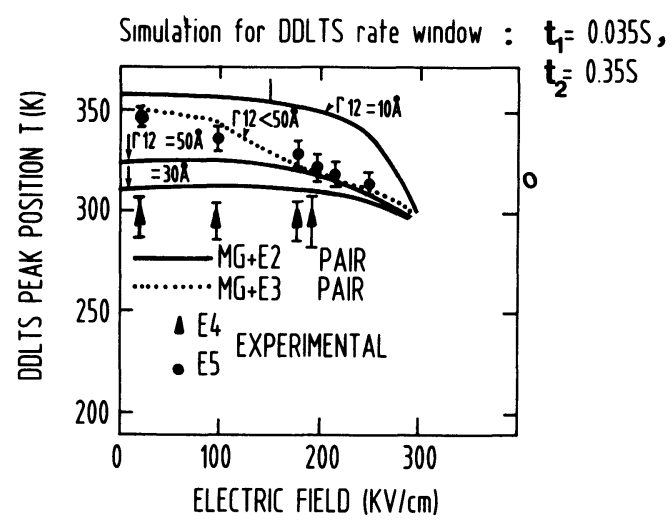

Fig. 5. - Model simulation for the Differential DLTS (DDLTS) peak position as a function of electric field for two types of defect-pair. The theory is compared with our own (LEP) DDLTS experimental results for the electronbombardment defects E4 and E5. The most significant uncertainty of the model is that of the DDLTS peak position predicted for the (MG + E2) pairs and is about $\pm 10 \mathrm{~K}$. For this pair, a minimum DDLTS peak temperature is predicted for $r_{12} \simeq 30 \AA$.

Different predictions are made for the pair $(\mathrm{MG}+\mathrm{E} 3)$ because E3 has a deeper level than that of E2 and equation (9a) gives a good approximation to the pair's electron emission rate. In figure 5 , we see that the DDLTS peak position of E5 and its high sensitivity to the electric field agree with those expected for the (MG + E3) pair. For this pair, the ionization rate $e_{n}$ obtained from equation (9a), is proportional - at different electric fields - to that $e_{2}$ of $\mathrm{E} 3$ which is known to be very sensitive to the electric field [28]. In contrast to the simulations for $(M G+E 2)$, those for $(M G+E 3)$ pairs proved practically insensitive to the pre-exponential factors of the model. 


\section{Interpretation of DLTS data in heavy-particle bombarded GaAs.}

We now make use of section 6 to interpret our DDLTS results for boron-ion implanted n-GaAs. We assume the densely packed defects to be MG, E3 and E2. The dotted curve in figure 6 corresponds to the well defined clusters approximation (Eq. (15)) with the same deep-level concentration ratio as for electron-irradiated materials $N_{1}: N_{2}: N_{3}=$ [MG]: $[\mathrm{E} 3]:[\mathrm{E} 2]=1: 2: 8$. The compensation ratio $\left(1-f_{1}\right)$ which enters equation (14) has been taken to be 0.5 in accordance with section 3 . Without any further parameter adjustment, the model predicts very well the observed DDLTS peak positions and their electric field dependence for the non-annealed samples.

The dashed curve of figure 6 corresponds to a simulation for uniformily and densely packed defects (Eq. (14)) with $N_{1}: N_{2}: N_{3}=[\mathrm{MG}]:$ [E3]: $[\mathrm{E} 2]=1: 1: 1$. This is seen to agree very well with experimental results for the sample annealed at $450{ }^{\circ} \mathrm{C}$.

More generally, for the samples annealed at increasingly higher temperatures, good agreement with experimental results is obtained if one introduces the two following assumptions in our computer simulations :

i) That the defects tend to be more uniformily distributed as the annealing temperature increases.

ii) That the ratio of shallow to deep level concen-

DDLTS rate window $t_{1}=35 \mathrm{~ms}, t_{2}=350 \mathrm{~ms}$

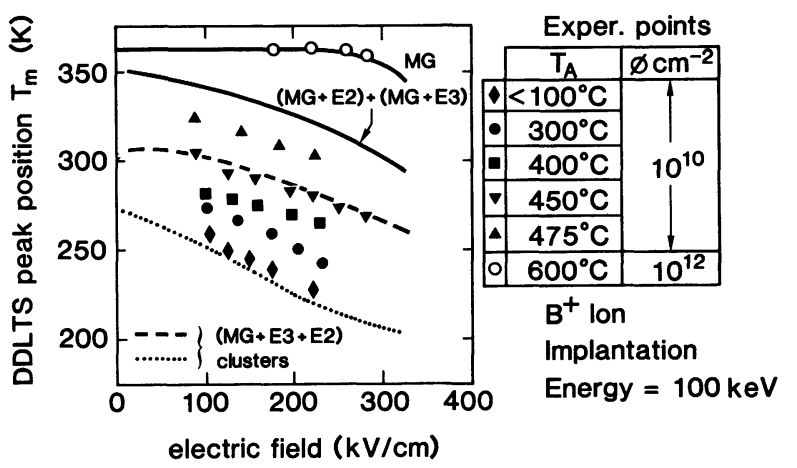

Fig. 6. - DDLTS peak positions versus junction electricfield. The dotted curve correspond to DDLTS model simulation for defects grouped in well defined clusters with $f_{1}=1 / 2$ and $N_{1}: N_{2}: N_{3}=1: 2: 8$. The dashed curve correspond to DDLTS model simulation for uniformily distributed densely packed defects with $f_{1}=1 / 2$ and $N_{1}: N_{2}: N_{3}=1: 1: 1$. The upper continuous curves correspond to isolated MG defects with parameters taken identical to those of EL2. The lower continuous curve corresponds to a combination in equal concentrations of the defect pairs $\mathrm{MG}+\mathrm{E} 2$ and $\mathrm{MG}+\mathrm{E} 3$. The experimental points correspond to ion-implantation defects for different $30 \mathrm{~min}$ isochronal annealing temperatures from reference [26]. tration decreases with increasing annealing temperature.

Inside a cluster, one expects a higher annealing rate than at its periphery since we know from earlier studies that long range recombination between implantation defects have an important influence [50]. Hence the defects will tend to become uniformily distributed in accordance to assumption (i). Assumption (ii) is confirmed by the fact that after ion implantation and annealing at $600{ }^{\circ} \mathrm{C}$, the only surviving irradiation induced electron-trap DLTS peak can be identified with the native mid-gap level EL2 [1, 51]. Both this level and EL2 are insensitive to the electric field in the range reported in figure 6 . One thus deduces that the MG defects anneal less quickly than E2 or E3 at temperatures up to $600^{\circ} \mathrm{C}$.

Our DLTS measurements indicate that the peak E3 (see Fig. 1) survives 30 min annealing steps up to $450{ }^{\circ} \mathrm{C}$. There is an apparent increase in the ratio of shallow to deep level concentrations which must be attributed to the reduction of the defect packing density (Fermi level not pinned anymore as in Fig. 4). In contrast, the annealing of E3 occurs at much lower temperatures in electron-irradiated $\mathrm{n}-\mathrm{GaAs}$. One reason for the E3 survival at higher anneal temperatures could be the charge state dependence of its annealing rate. Pons [52] has demonstrated that the annealing rate of an ionized E3 defects is much smaller than for those occupied with electrons. Using the data of this author, one can extrapolate the annealing rates up to temperatures in the vicinity of $400{ }^{\circ} \mathrm{C}$ for unoccupied E3 defects (as expected near a defect cluster in Fig. 4). This would predict an annealing stage for $\mathrm{E} 3$ near to $350{ }^{\circ} \mathrm{C}$ (instead of above $400^{\circ} \mathrm{C}$ ). The discrepancy between expected and observed annealing is probably due to defectinduced changes in the Debye temperature of the material. The level E2 in electron-irradiated material has a more reduced charge-state dependence than E3 for its thermal annealing rate [52] so one may assume that the E2 concentration in the clusters decreases faster than that of E3 after annealing. In samples annealed above $450^{\circ} \mathrm{C}$ the defect density decreases to such an extent that inequalities (13) are expected to fail. It is therefore understandable why the DDLTS peaks behave as a mixture of densely packed defects combined with isolated $(M G+E 2)$ and $(\mathrm{MG}+\mathrm{E} 3)$ pairs.

In the framework of our computer simulations, the observed behaviour of the DDLTS peaks for different electric fields and annealing treatments is consistent with the above-reviewed annealing properties of the E2, E3 and MG defects.

\section{Nature of the irradiation-induced defects.}

As discussed in sections 2.1, experimental evidence for electron-irradiated n-GaAs leads us to attribute 
the high-temperature DLTS peaks E4 and E5 to the defect pairs $(M G+E 2)$ and $(M G+E 3)$ respectively. As was shown in section 7, our calculations for such pairs confirm these attributions. Furthermore, identifying E5 with the pair (MG + E3) is consistent with the fact that E5 and E3 are observed to show very similar thermal [21] and recombination enhanced [22] annealing behaviour over a wide temperature range.

Both E2 and E3 are very probably associated with an As vacancy [53]. This suggests that E4 and E5 may be viewed as the result of the initial formation of the defect pair $\mathrm{V}_{\mathrm{Ga}}-\mathrm{Ga}_{i}$ as part of a primary Frenckel defect. This pair is believed to be unstable $[21,54,55]$; it may either recombine or else a nearest neighbour As lattice atom can move into the Ga vacancy, thus producing two stable defects $A s_{G a}$ and $V_{A s}$ which may subsequently diffuse apart. The observation by EPR of the corresponding «precursor» defect complex $\left(\mathrm{As}_{\mathrm{Ga}} \mathrm{V}_{\mathrm{As}}\right)$ has been recently reported by von Bardeleben et al. [54, 55]. The electron irradiating beam does not only induce lattice ion displacements, it is also expected to have a strong ionizing influence and to liberate considerable amounts of phonon energy. As a consequence, one expects the mobility of the crystal's As and Ga interstitial atoms to be greatly enhanced during irradiation [21]. These diffusing interstitial atoms can then be preferentially trapped at $V_{A s}$ or $\mathrm{As}_{\mathrm{Ga}}$ related interstitial sites. Details of this defect creation process remain to be established by further studies.

In interpreting the electrical conductivity observations in bombarded GaAs in sections 2 and 3 it appeared unavoidable to invoke a mid-gap irradiation-induced defect (MG). In samples annealed below $600{ }^{\circ} \mathrm{C}$, this MG level is not directly observable by DLTS. In sections 5 to 8 the observed hightemperature peaks were, however, accounted for either by defect pairs including MG together with a shallower defect (in fast-electron-irradiated $\mathrm{n}-\mathrm{GaAs}$ ) or by damage clusters including MG, E2 and E3 defects (in heavy-particle bombarded nGaAs). For heavy-particle bombardment, we showed in section 6.2 , that only the defects at the clusters' outskirts have an appreciable influence on DC electrical conductivity and DLTS observations. The ion bombardment damage energy at these outskirts is comparable to the damage energy due to fast-electron irradiation. This explains why we were able to attribute results in heavy-particle and electron-irradiated materials to the same types of individual point defects.

The MG defect (irradiation induced) and the EL2 defect (native in bulk GaAs) share the properties of both being single donors, having mid-gap energy levels and being associated with the $\mathrm{As}_{\mathrm{Ga}}$ defect. Furthermore, we have shown in section 3 that the lattice relaxation parameters $\hbar \omega$ and $S \hbar \omega$ for the two defects are similar. One is thus led to identify MG with the deep level associated to EL2.

A widely accepted signature revealing the presence of EL2 defects is the " quenching » behaviour observed in the photo-capacitance transients corresponding to sub-band-gap illumination $[1,51]$. The occurrence of this behaviour in heavy-ion bombarded materials subsequently annealed above $600{ }^{\circ} \mathrm{C}$ gives clear evidence for the creation of EL2 $[51,1]$. For samples annealed below $600{ }^{\circ} \mathrm{C}$, the absence of the «quenching » behaviour is often used as an argument to demonstrate that EL2 is not created during ion bombardment [25]. In our present work we were led to believe that all the irradiationinduced defects MG are part of defect pairs or dense damage clusters for samples annealed below $600^{\circ} \mathrm{C}$. Consequently, although MG and EL2 are probably identical, it is easy to understand the observed departure of the photocapacitance transients of MG defects from the transients typical of isolated EL2 defects.

\section{Summary and conclusions.}

Making use of a recently developed theory, we have studied the multi-phonon assisted hopping DC conductivity in GaAs induced by heavy particle bombardment. From our best-fit procedure, we deduced that the irradiation induced mid-gap (MG) level can be identified with that of the native EL2 centre.

We have also mathematically simulated the Deep Level Transient Spectroscopy (DLTS) response of defect pairs and clusters taking in account the hopping of electrons between the individual defects involved. We attributed each of the high-temperature DLTS peaks E4 and E5 in fast-electron bombarded $\mathrm{n}-\mathrm{GaAs}$ to distant defect-pairs involving the $\mathrm{As}_{\mathrm{Ga}}$ anti-site related donor level MG together with shallower $V_{\text {As }}$ related acceptor defects. The hightemperature DLTS peak in heavy-particle bombarded materials is accounted for by our simulations for defect clusters. For defect pairs as well as clusters, we account for the observed wide DLTS peaks, together with their often high sensitivity to the electric field. Our DLTS studies confirm that EL2 and MG are related.

\section{Acknowledgments.}

The theoretical background to this work has been partly developed in collaboration with M. Lannoo. The authors wish to thank P. Langlade who has performed part of the electrical measurements. Thanks are also due to $\mathbf{H}$. J. von Bardeleben, H. M. J. Boots, J. C. Bourgoin, R. Eppenga, L. F. Feiner, A. Goltzene, F. J. A. Greidanus, J. C. M. Henning, S. Kalbitzer, H. W. van Kesteren, 
E. Montie, D. Pons, A. Roizes, M. F. H. Schuurmans and D. Stievenard for fruitful discussions. We acknowledge skilful technical assistance from E. Boucherez and M. Auger.

\section{References}

[1] Makram-Ebeid, S., Langlade, P. and Martin, G. M., in Semi-Insulating III-V Materials 1984, Ed. D. C. Look and J. S. Blakemore (Shiva, Nantwich) 1984, pp. 184-203.

Martin, G. M. and MaKram-Ebeid, S., in Deep Centers in Semiconductors: A State-of-the-Art Approach Ed. Sokrates Pantelides (GordonBreach Science Publ., N.-Y.) 1986, pp. 399-487.

[2] Lagowski, J. and Gatos, H. C., in Thirteenth International Conference on Defects in Semiconductors, Ed. L. C. Kimerling and J. M. Parsey Jr. (Metallurgical Society of AIME) 1985, pp. 73-86.

[3] SCHNEIDER, J., in Semi-Insulating III-V Materials 1982, Ed. S. Makram-Ebeid and B. Tuck (Shiva, Nantwich) 1982, p. 144.

[4] Von Bardeleben, H. J., Stievenard, D., Huber, A. and Bourgoin, J. C., Phys. Rev. B 34 (1986) 7191.

[5] Figielski, T. and Wosinski, T., Phys. Rev. B 36 (1987) 1269.

Meyer, B. K., Hofmann, D. M., Niklas, J. R. and Spaeth, J.-M., Phys. Rev. B 36 (1987) 1332.

Levinson, M. and Kafalas, J. A., Phys. Rev. B 35 (1987) 9383.

[6] Goltzéné, A., Schwab, C., David, J. P. and RoIzès, A., Appl. Phys. Lett. 49 (1986) 862.

[7] RoIzÈs, A., private communication.

[8] Hasegawa, H. and Sawada, T., Thin Solid Films 103 (1983) 119.

[9] Makram-Ebeid, S., Boher, P. and Lannoo, M., J. Appl. Phys. (May 1988).

[10] Makram-Ebeid, S. and Lannoo, M., Phys. Rev. B 25 (1982) 6406.

[11] Makram-Ebeid, S. and Lannoo, M., Phys. Rev. Lett. 48 (1982) 1281.

[12] Bebs, H. B. and Chapman, R. A., in Proc. of the 3rd International Conf. on Photoconductivity, Ed. E. M. Pell (Pergamon Press, New York) 1971 , p. 245.

[13] Kane, E. O., J. Phys. Chem. Solids 1 (1957) 249.

Kane, E. O., J. Phys. Chem. Solids 12 (1959) 181.

[14] Abramowitz, M. and STEgun, I. A., Handbook of Mathematical Functions (Dover Publications, New York) 1965.

[15] Burt, M., J. Phys. C 13 (1980) 1825.

[16] LANG, D. V., in Radiation Effects in Semiconductors 1976, Inst. Phys. Conf. Ser. 31 (1977) 70-85.

[17] SeITZ, F., Discuss. Faraday Soc. 5 (1949) 271.

[18] VAN Lint, V. A. J. and LEAdon, R. E., in GaAs and Related Compounds 1974, Inst. Phys. Conf. Ser. 23 (1975) 227-232.

[19] BricE, D. K. (volume 1) and Winterbon, K. B. (volume 2), Ion Implantation ranges and Energy
Deposition Distributions (IFI/Plenum, New York) 1975.

[20] BARnes, C. E., ZipPERIAN, T. E. and DAwson, L. R., J. Electron. Mater. 14 (1985) 95.

[21] Pons, D. and Bourgoin, J. C., J. Phys. C 18 (1985) 3839.

[22] Stievenard, D. and Bourgoin, J. C., J. Appl. Phys. 59 (1986) 808.

[23] Lefèvre, H. and Schulz, M., Appl. Phys. 12 (1977) 45.

[24] Goswami, N. K., Newman, R. C. and Whitehouse, J. E., Solid State Commun. 40 (1981) 473.

[25] Von Bardeleben, H. J. Stievenard, D., Hubert, A. and Bourgoin, J. C., Appl. Phys. Lett. 47 (1985) 970.

[26] Langlade, P. and Makram-Ebeid, S., in GaAs and Related Compounds 1984 Inst. Phys. Conf. Ser. 74 (1985).

[27] LANGLADE, P., unpublished results.

[28] Makram-Ebeid, S., Appl. Phys. Lett. 37 (1980) 464.

[29] Thurmond, C. D., J. Electrochem. Soc. 122 (1975) 1133.

[30] Mircea, A., Mitonneau, A. and Vannimenus, J., J. Phys. Lett. France 38 (1977) L 41.

[31] Pons, D., Paget, D. and Kaplan, D. (1985), Final Contract Report (Number MRT 82 A 1481) to the French Ministry of Industry and Research. The part of the data on trapping kinetics of E1 can be found in D. Pons and J. C. Bourgoin ; same Proc. as reference 2, pp. 989-995.

[32] Pons, D., Mircea, A. and Bourgoin, J. C., J. Appl. Phys. 51 (1980) 4150.

[33] Chantre, A., Vincent, G. and Bois, D., Phys. Rev. B 23 (1981) 5335.

[34] Deng, X. C., LiU, X. H., Boehringer, K. and Kalbitzer, S., Appl. Phys. A 33 (1984) 29.

[35] MotT, N. F. and Davis, E. A., Electronic Processes in Non-Crystalline Materials (Clarendon Press, Oxford) 1979.

[36] Coates, R. and Mitchell, E. W. J., Adv. Phys. 24 (1975) 593.

[37] Miller, A. and Abrahams, E., Phys. Rev. 120 (1960) 7745.

[38] Pike, G. E. and Seager, C. H., Phys. Rev. B 10 (1974) 1421.

[39] Butcher, P. N., Philos. Mag. B 42 (1980) 799.

[40] Makram-Ebeid, S., Boher, P., LANNoo, M., Appl. Phys. Lett. 50 (1987) 270-272.

Makram-Ebeid, S. and Boher, P., in Proc. of the 14th Internat. Conf. on Defects in Semiconductors (Mat. Science Forum vol. 10-12, Trans. Tech. Publ. Ltd Switz.) 1986, pp. 1075-1080.

[41] For a review see: JoNSCHER, A. K., Nature 256 (1975) 566. 
[42] Pollak, M. and Geballe, T. H., Phys. Rev. 122 (1961) 1742.

[43] Mcinnes, J. A., Butcher, P. N. and Clark, J. D., Philos. Mag. B 41 (1980) 1.

[44] Margenau, H. and Lewis, M., Rev. Mod. Phys. 31 (1959) 569.

[45] Mareš, J. J., KristofiK, J., Šmíd, V., Zeman, J. and ŚtouraČ, L., in Semi-Insulating III-V Materials, Ed. H. Kukimoto and S. Miyazawa (Ohmsha/North-Holland, Tokyo) 1986, pp. 127132.

[46] Courtney, W. E., IEEE Trans. Microwave Theory Tech. MTT-25 (1977) 697.

[47] Rees, G. J., Grimmeis, H. G., Janzen, E. and Skarstam, B., J. Phys. C 13 (1980) 6157.

[48] Pons, D. and Makram-EbeId, S., J. Phys. France 40 (1979) 1161.

[49] Stievenard, D., Lannoo, M. and Bourgoin, J. C., Solid-State Electron. 28 (1985) 485.
[50] Martin, G. M., Esteve, E., Langlade, P. and Makram-Ebeid, S., J. Appl. Phys. 56 (1984) 2655.

[51] Taniguchi, M. and Iкoma, T., Appl. Phys. Lett. 45 (1984) 69.

[52] Pons, D., in Defects and Radiation Defects in Semiconductors 1980, Inst. Phys. Conf. Ser. 59 (1981) 269-274.

[53] Loualliche, S., Nouilhat, A., Guillot, G. and LANNOO, M., in Thirteenth International Conference on Defects in Semiconductors, Ed. L. C. Kimerling and J. M. Parsey Jr. (Metallurgical Society of AIME) 1985, pp. 967-971.

[54] Von Bardeleben, H. J., Bourgoin, J. C. and Miret, A., Phys. Rev. B 34 (1986) 1360.

[55] Von Bardeleben, H. J., Miret, A., Lim, H. and Bourgoin, J. C., J. Phys. C 20 (1987) 1353. 